Supporting Information

\title{
Mixed ionic and electronic conduction in radical polymers
}

\author{
Ilhwan Yu, ${ }^{1}$ Daeyoung Jeon, ${ }^{1}$ Bryan Boudouris, ${ }^{2,3}$ Yongho Joo ${ }^{1, *}$
}

${ }^{1}$ Institute of Advanced Composite Materials, Korea Institute of Science and Technology (KIST), 92 Chudong-ro, Bongdong-eup, Wanju-gun, Jeonbuk, 55324, Republic of Korea

${ }^{2}$ Charles D. Davidson School of Chemical Engineering, 480 Stadium Mall Drive, Purdue University, West Lafayette, IN 47906, United States.

${ }^{3}$ Department of Chemistry, 560 Oval Drive, Purdue University, West Lafayette, IN 47906, United States.

\section{Materials}

All chemicals were purchased from Sigma-Aldrich (United State America) unless stated otherwise. The (2,2,6,6-tetramethylpiperidin-1-yl)oxidanyl (TEMPO) was purchased from TCI Tokyo. The solvents, tetrahydrofuran (THF) and hexane (HPLC grade), were obtained from Daejung Co. Korea. Sodium hydroxide $(\mathrm{NaOH}$, analytical grade) was obtained from TCI Tokyo.

\section{PTEO Synthesis}

The 4-glycidyloxy-2,2,6,6-tetramethylpiperidine-1-oxyl (gTEMPO) was synthesized by adopting a previously reported procedure for synthesis. $\left.{ }^{[1,} 2\right]$ A mixture of the tetrabutylammonium hydrosulfate $(1.5 \mathrm{~g}, 4.6 \mathrm{mmol})$ and epichlorohydrin $(10 \mathrm{~mL}, 120 \mathrm{mmol})$ was dissolved in aqueous sodium hydroxide solution $(30 \mathrm{~mL}, 50 \mathrm{wt} \%)$. After stirring, a solution of 4-hydroxy-2,2,6,6-tetramethylpiperidin-N-oxy (4.12 g, $23 \mathrm{mmol})$ dissolved in tetrahydrofuran $(200 \mathrm{~mL})$ was added as droplets and the solution stirred overnight at room temperature. Following this, the reactants were dropped in ice water $(300 \mathrm{~mL})$ and extracted with ethyl acetate. The organic solution was gathered and the water was removed using 
anhydrous magnesium sulfate. The anhydrous magnesium sulfate was filtered and the organic solvent was evaporated through the rotary evaporator to produce the crude product. The crude oil was purified using column chromatography (silica gel) with an organic solvent (hexane: ethyl ether $=8: 1, \mathrm{v} / \mathrm{v}$ ) as an eluent. The solution was evaporated through the rotary evaporator, and red crude oil was recrystallized with hexane to obtain isolated red solid (Yield: 87\%).

A mixture of gTEMPO (1 g, $4.4 \mathrm{mmol})$ and potassium tert-butoxide $(20 \mathrm{mg}, 0.18$ mmol) was sealed in a glass vial inside a glove box. ${ }^{[3]}$ The mixture was heated at $80{ }^{\circ} \mathrm{C}$ for $2 \mathrm{~h}$ in the glove box. After cooling, dichloromethane was added to the reaction mixture, and the organic solution was washed with deionized water. After that, the separated organic layer was mixed with hexanes, producing an oily, viscous liquid. This viscous product of polymer was dissolved in tetrahydrofuran, and the polymer was precipitated in hexanes. The polymer was dried for 12 hours in a vacuum oven at room temperature. The number-average molecular weight $\left(\mathrm{M}_{\mathrm{n}}\right)$ of produced poly(4-glycidyloxy-2,2,6,6-tetramethylpiperidine-1-oxyl) (PTEO) was $1.3 \mathrm{~kg} \mathrm{~mol}^{-1}$, with a dispersity value (Đ) of 1.35 , as measured using gel permeation chromatography (GPC) using polystyrene standards. The radical density of PTEO characterized by the EPR was $98 \%$.

\section{General Methods}

Differential scanning calorimetry (DSC) analysis was measured using a TA Instruments STDQ600 TGA-DSC. The polymer and polymer-ion mixture samples were initially heated to $90{ }^{\circ} \mathrm{C}$ held isothermally for $5 \mathrm{~min}$, and then cooled to $-50{ }^{\circ} \mathrm{C}$ under a nitrogen gas purge. All of the heating and cooling ramps occurred at a rate of $10{ }^{\circ} \mathrm{C} \mathrm{min}^{-1}$. Then, the sample was exposed to the same procedure for a second heating and second cooling scan. The data shown are from the second scans of heating. Thermogravimetric (TGA) analysis data were collected through a 
Discovery TGA 55 (TA instrument). To analyze the degradation temperature, the polymer and polymer ion mixture samples were heated from $5{ }^{\circ} \mathrm{C}$ to $500{ }^{\circ} \mathrm{C}$ at a scan rate of $5{ }^{\circ} \mathrm{C} \mathrm{min}^{-1}$ under air. For the thermal stability analysis, the sample was annealed at $40{ }^{\circ} \mathrm{C}$ for $10 \mathrm{~min}$ under the vacuum chamber. Electron paramagnetic resonance (EPR) spectroscopy experiments were completed using a Bruker EMX-EPR spectrometer. For the solution EPR experiments, the PTEO and LiTFSI mixture was dissolved in chloroform and placed in the EPR tubes. After that, the EPR tubes were placed in the holder, and held at a given temperature for 10 minutes to allow for the thermal equilibration. The EPR data were collected at room temperature.

Current-voltage $(\mathrm{I}-\mathrm{V})$ measurements were analyzed by sweeping voltages in the range of $-1 \mathrm{~V}$ $\leq V \leq+1 \mathrm{~V}$ or $-10 \mathrm{~V} \leq V \leq+10 \mathrm{~V}$ with Keithley 4000 source meter (A Tektronix company, United States). These data were recorded with the Lab Tracer software (A Tektronix company, United States). The optical transmittance of the PTEO and the PTEO-ions was measured using a UV-VIS-NIR spectrophotometer (V-670, Jasco Corporation, Tokyo, Japan) in the range of 300-800 nm. For the UV-VIS spectroscopy, 4-hydroxy-TEMPO and polymer (PTEO) samples were dissolved into the THF with a concentration of $1 \mathrm{mg} \mathrm{mL}^{-1}$. The solution absorption and thin-film transmission values were monitored between wavelengths of $300 \mathrm{~nm} \leq \lambda \leq 800 \mathrm{~nm}$. XPS analyses were carried out using a Thermo Scientific K-Alpha (Thermo Fisher Scientific Co., United States) using $400 \mu \mathrm{m}$ spot size with an X-ray photoelectron spectrometer system. The X-ray beam source was Al Ka micro-focused monochromator. The thin films of the samples used for XPS was prepared on a silicon wafer substrate, and the films were spun-coat to obtain uniform thicknesses across the samples. The Fourier transform infrared (FT-IR) spectroscopy data were collected by using a ATR-FTIR spectrophotometer (Jasco Co., Tokyo, Japan, FT/IR-6600 model).

\section{Device characterization}


Glass substrates were cleaned with a piranha solution $\left(\mathrm{H}_{2} \mathrm{O}_{2}: \mathrm{H}_{2} \mathrm{SO}_{4}=33 \%: 67 \%\right.$, by volume) for 30 minutes and then rinsed with deionized water to remove any surface residue. After cleaning, $\mathrm{Cr} / \mathrm{Au}(10 / 50 \mathrm{~nm})$ electrodes were deposited on a glass slide using a shadow mask using a thermal evaporator at the pressure of $1 \times 10^{-6}$ bar to create the bottom contact electrodes on a glass substrate. The channel length $\left(L_{c h}\right)$ and the channel width $\left(W_{c h}\right)$ of the device were $50 \mu \mathrm{m}$ and $1 \mathrm{~cm}$, respectively. For the channel length-dependent conductivity characterization, the channel lengths ranged from 2 to $50 \mu \mathrm{m}$, and these were fashioned using standard photolithography techniques. After device fabrication, the PTEO and PTEO-LiTFSI solutions in THF with a concentration of $1 \mathrm{mg} \mathrm{mL}^{-1}$ was drop-casted onto the device crossing the two electrodes, yielding uniform films with a thickness of $\sim 50 \mu \mathrm{m}$, as determined using a P7 profilometer. The PTEO-LiTFSI was mixed in the glove box to minimize the moisture contamination.

Current-voltage (I-V) measurements were collected using sweeping voltages in the range of -1 $\mathrm{V} \leq \mathrm{V} \leq+1 \mathrm{~V}$ or $-10 \mathrm{~V} \leq \mathrm{V} \leq+10 \mathrm{~V}$ for device channel lengths of $2 \mu \mathrm{m}, 3 \mu \mathrm{m}, 4 \mu \mathrm{m}, 5 \mu \mathrm{m}$, $10 \mu \mathrm{m}$, and $50 \mu \mathrm{m}$. These data were recorded and analyzed using Lab Tracer software. From these data, the electrical conductivity was calculated using Ohm's law. These experiments were performed under vacuum in a home-built probe station with a Keithley 2400 source meter, for the recording and analysis of I-V sweep. The temperature-dependent electrical conductivity variations were also recorded by the same probe station under vacuum condition $\left(1 \times 10^{-6}\right.$ bar $)$ in the temperature range of $100 \mathrm{~K}<\mathrm{T}<400 \mathrm{~K}$. The combination of liquid nitrogen and a temperature controller controlled the temperature of stages and the samples. Several control experiments were performed in ambient conditions at room temperature.

\section{AC Impedance Spectroscopy.}

The ionic conductivity was measured by HP4192A impedance analyzer in the range of $10 \mathrm{~Hz}-$ 
$10 \mathrm{MHz}$ at $50 \mathrm{mV}$ oscillator level with frequency response analyzer. The impedance measurements were taken upon heating between 20 and $50^{\circ} \mathrm{C}$. For the equilibrium condition, samples hold at each temperature for $10 \mathrm{~min}$. Two measurements were measured and averaged at a specific temperature for accuracy. The ionic conductivity was calculated as $\sigma=d /\left(R_{x} A\right)$, where the $\mathrm{Rx}$ is bulk resistance of electrolyte, $\sigma$ is an ionic conductivity, $\mathrm{d}$ is the distance between electrodes and $\mathrm{A}$ is the area between the electrolyte and electrode.
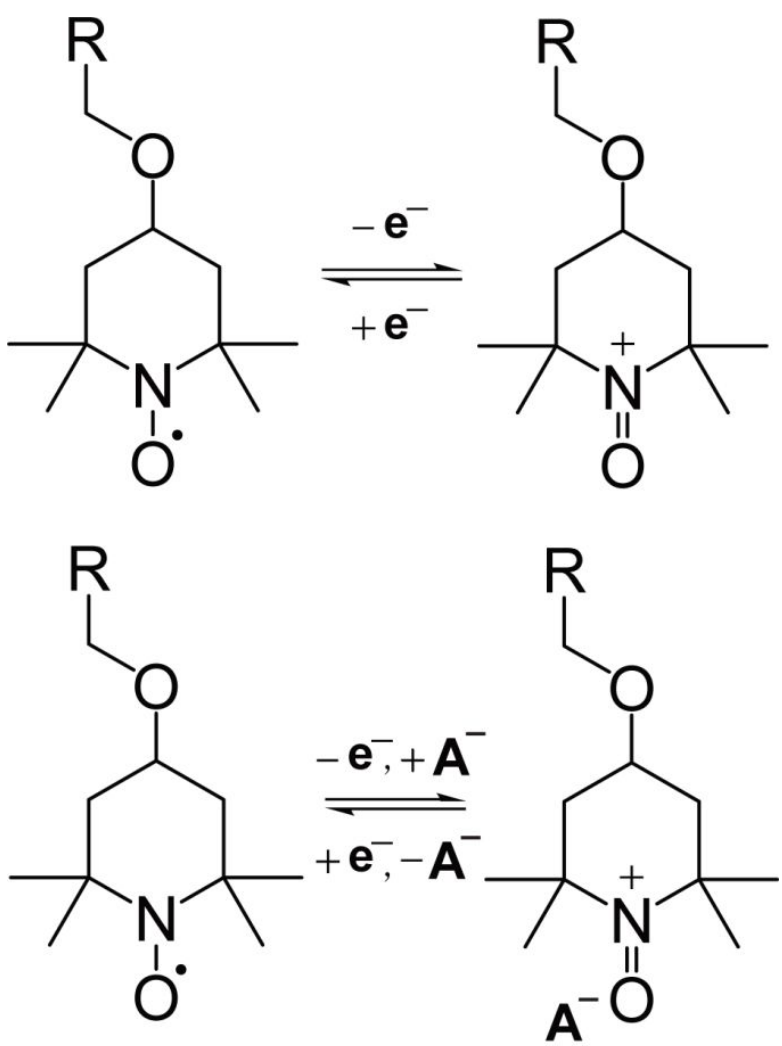

Figure S1. Schematic illustration of the PTEO redox reaction. PTEO undergoes oxidation by chemical doping, converting the neutral organic radical to an oxoammonium cation species. 


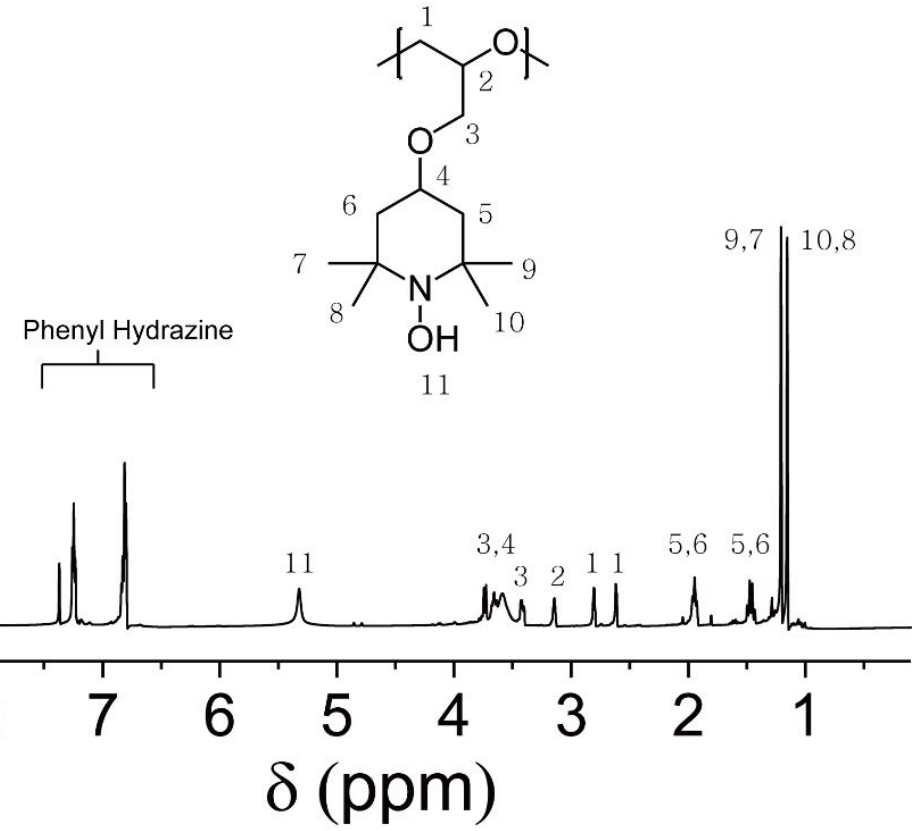

Figure S2. ${ }^{1} \mathrm{H}$ NMR of PTEO (chloroform-d) after quenching the radical with phenyl hydrazine.
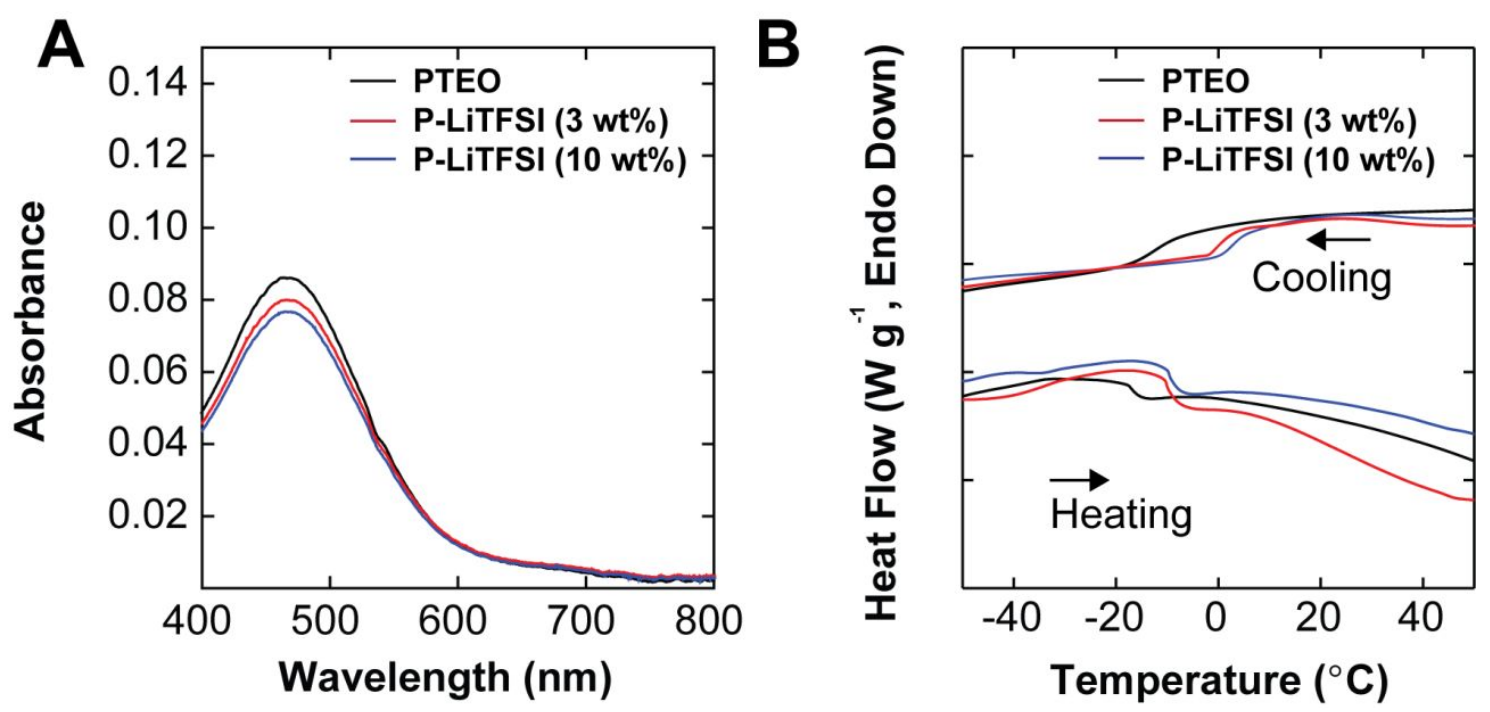

Figure S3. (A) UV-VIS absorption spectroscopy of PTEO illustrating the decrease of nitroxide group in thin films. (B) The glass transition temperature of PTEO and PTEO-LiFTSI (3 and 10 $\mathrm{wt} \%)$. 

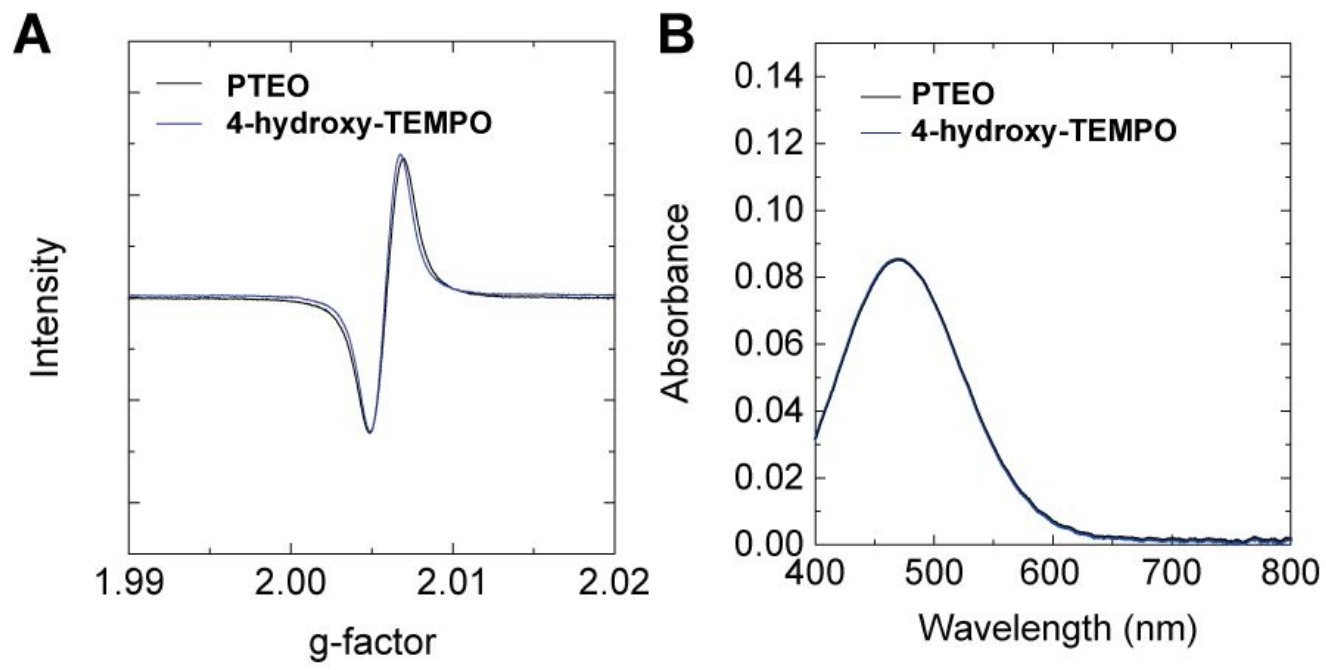

Figure S4. (A) Solid EPR (thickness $=50 \mu \mathrm{m}$ ) and (B) UV-VIS spectroscopy ( $1 \mathrm{mg} \mathrm{m}^{-1}$ at THF) of PTEO and 4-hydroxy TEMPO.

Table S1. The percentage of N-OH, N-O ${ }^{\circ}$ and +NO of PTEO and PTEO-LiTFSI composites examined by XPS.

\begin{tabular}{|l|l|l|l|}
\hline & $\mathrm{NOH}$ & $\mathrm{N}-\mathrm{O}^{\circ}$ & $+\mathrm{NO}$ \\
\hline PTEO & - & $100 \%$ & - \\
\hline PTEO-LiTFSI (3 $\mathrm{wt} \%)$ & $22 \%$ & $55 \%$ & $23 \%$ \\
\hline PTEO-LiTFSI $(10 \mathrm{wt} \%)$ & $27 \%$ & $48 \%$ & $25 \%$ \\
\hline
\end{tabular}




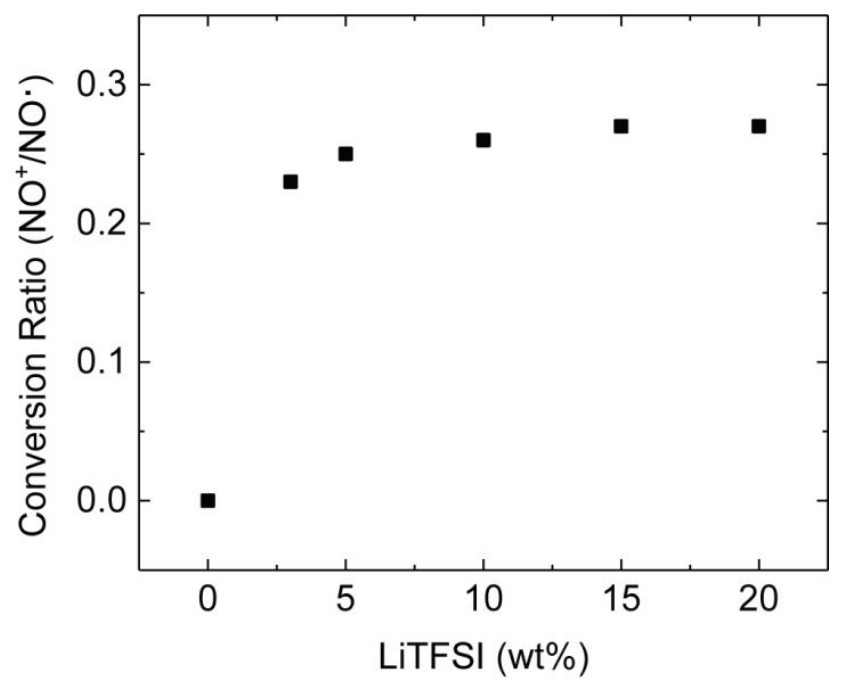

Figure S5. The relative conversion ratio from radical species (-NO ') to oxoammonium cationic species $\left(-\mathrm{NO}^{+}\right)$of PTEO radical polymer as a function of LiFTSI loading in a range of 0 to $20 \%$ (by weight). The conversion ratio was calculated by comparing the underneath area of $-\mathrm{NO}^{-}$and $-\mathrm{NO}^{+}$from XPS.

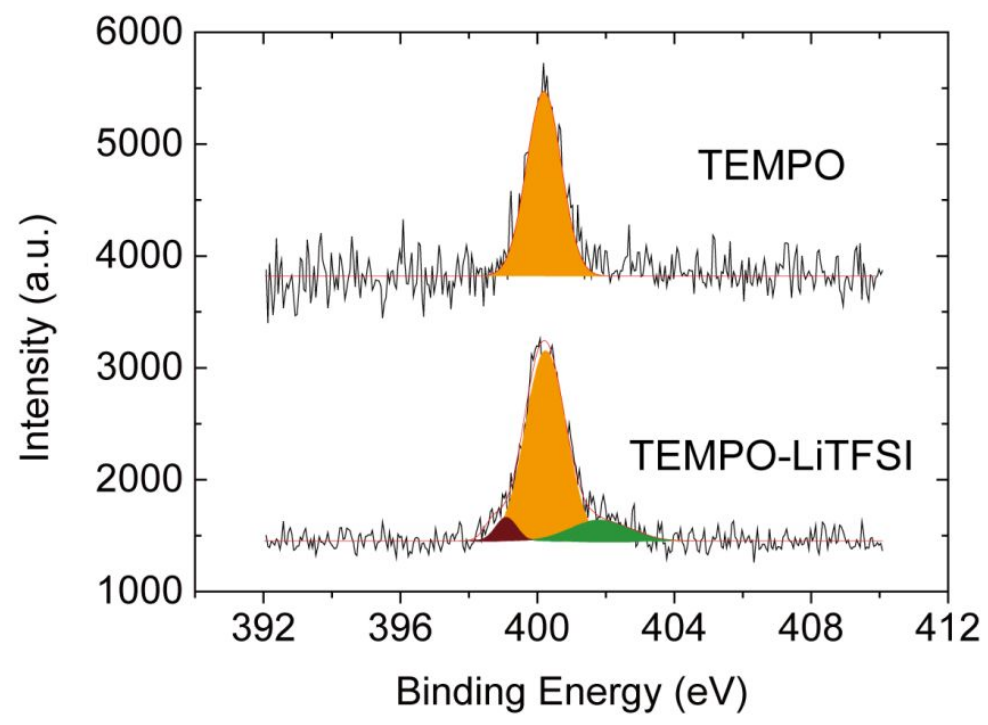

Figure S6. High magnification XPS spectra of the TEMPO-LiTFSI (10 wt $\%)$ thin films around the N1s peaks. 


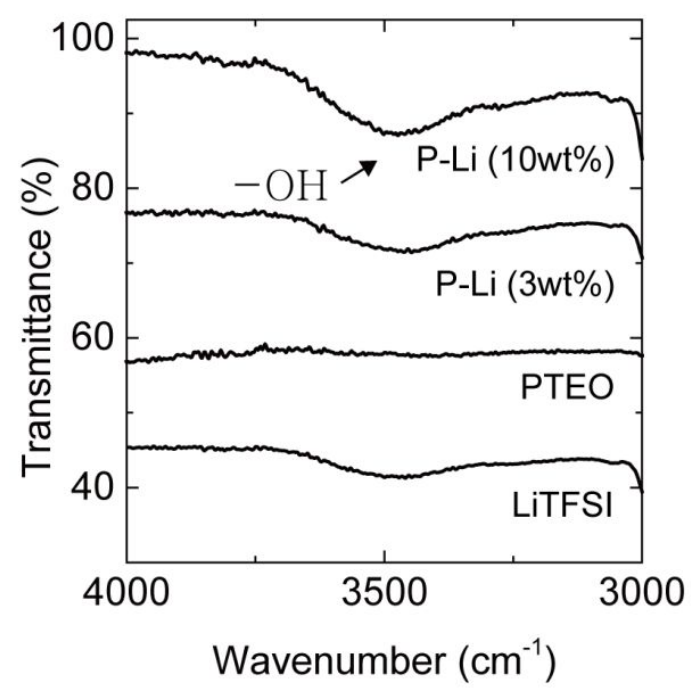

Figure S7. Magnified region of the ATR-FTIR spectra of PTEO, PTEO-LiTFSI $(3 \mathrm{wt} \%)$, PTEO-LiTFSI (10wt\%) and pristine LiTFSI shows the presence of the electricallyinsulating protonated pendant group species (i.e., N-OH). The intensity of the absorption increases slightly with increasing amounts of ions; this corresponds well with the disproportionation reaction that forms the insulating species. The pristine LITFSi contains the small amount of residual water.

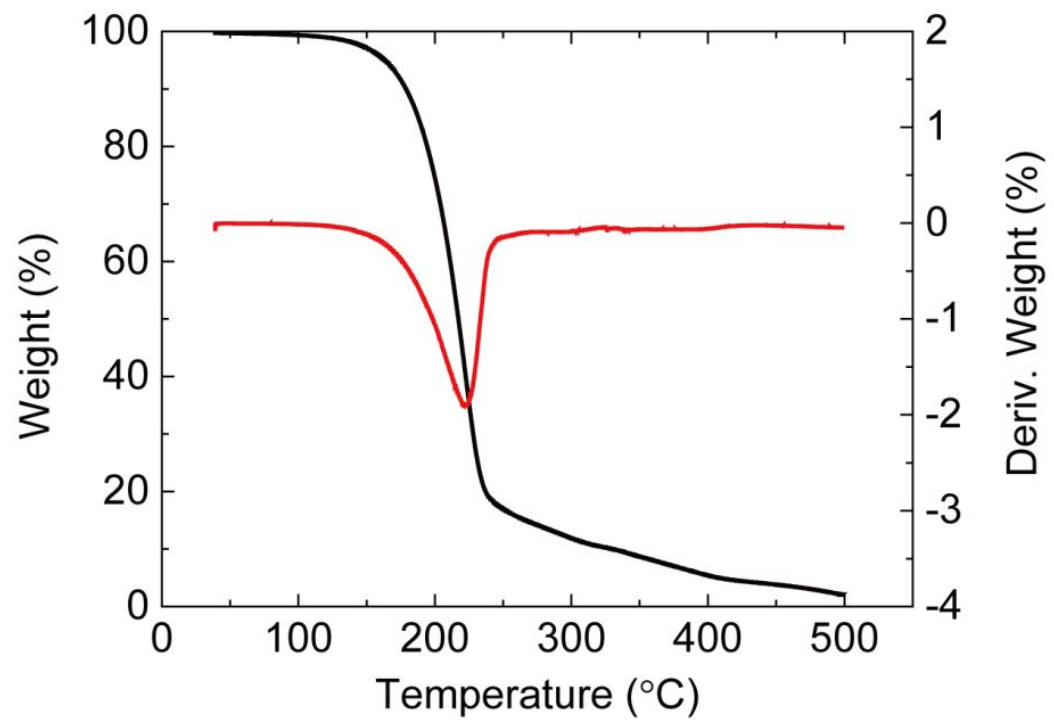

Figure S8. Thermogravimetric analysis of PTEO, showing the onset degradation temperature of PTEO $\sim 145^{\circ} \mathrm{C}$. 

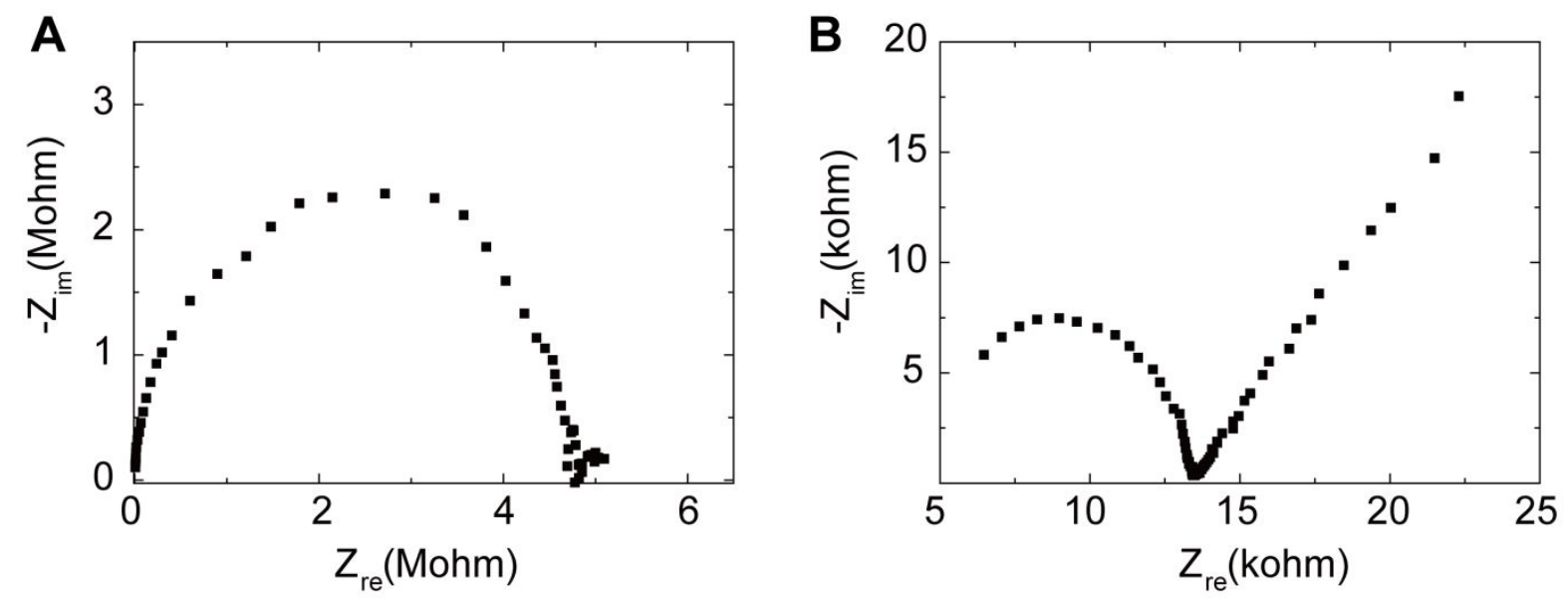

Figure S9. Representative AC impedance spectroscopy of PTEO-LiTFSI (10 wt \%) mixture. The ionic conductivity was measured in devices of $\mathrm{Cr} / \mathrm{Au}(10 / 50 \mathrm{~nm})$ electrodes on glass with the channel length $\left(L_{c h}\right)$ of $\sim 50 \mu \mathrm{m}$ and the channel width $\left(W_{c h}\right)$ of $1 \mathrm{~cm}$, respectively.

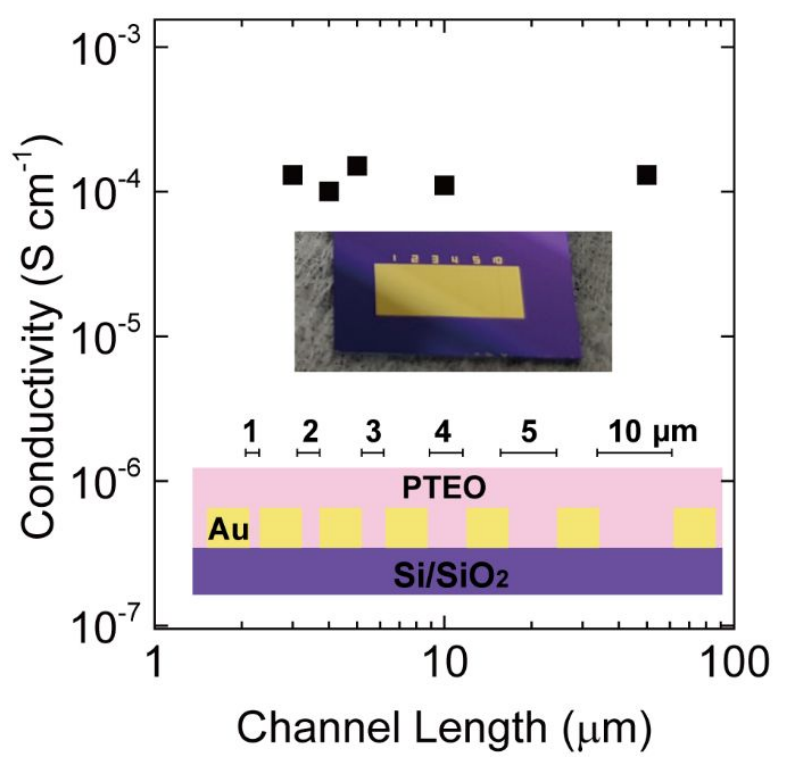

Figure S10. The mixed ionic and electronic conductivity as a function of channel length in a range of $1-50 \mu \mathrm{m}$, showing the minimal variation of conductivity. 


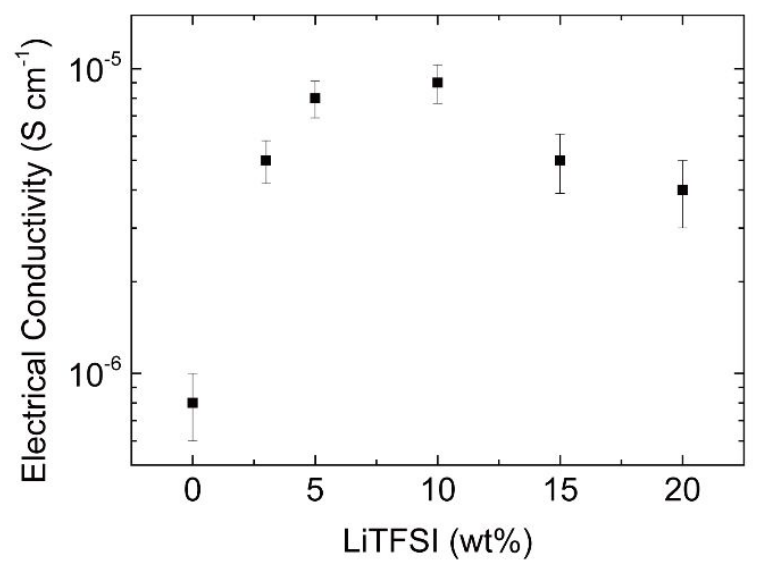

Figure S11. Electrical conductivity variation as a function of weight percentage of LiTFSI in PTEO.

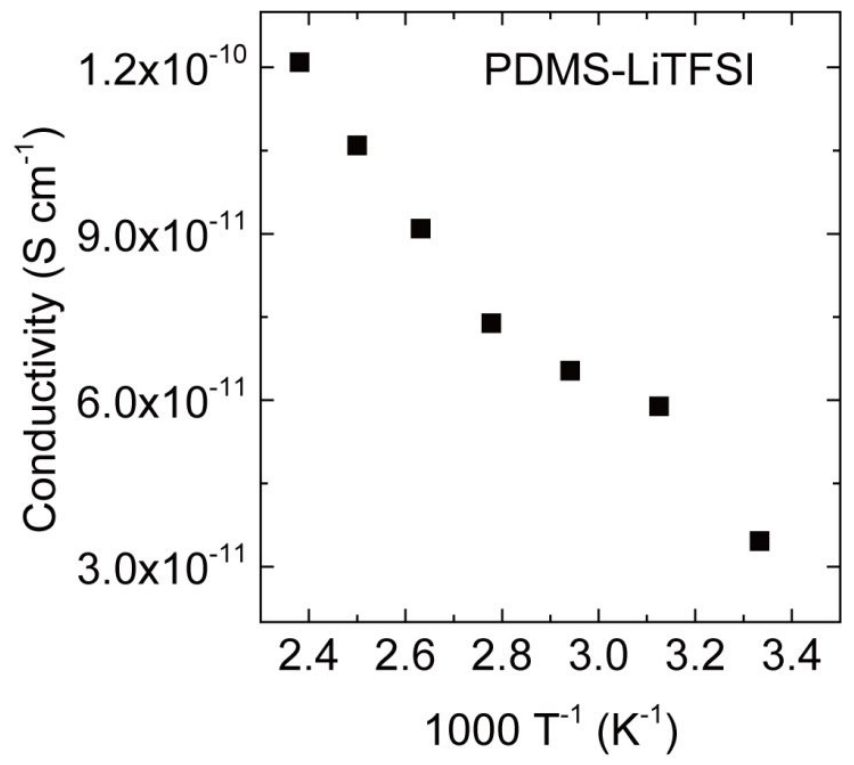

Figure S12. Plot for the conductivity variation of polydimethylsiloxane (PDMS) as a function of temperature (Channel length $50 \mu \mathrm{m}$, Thickness $=10 \mu \mathrm{m}$ ), showing the low conductivity (i.e., insulator-like) behavior. 


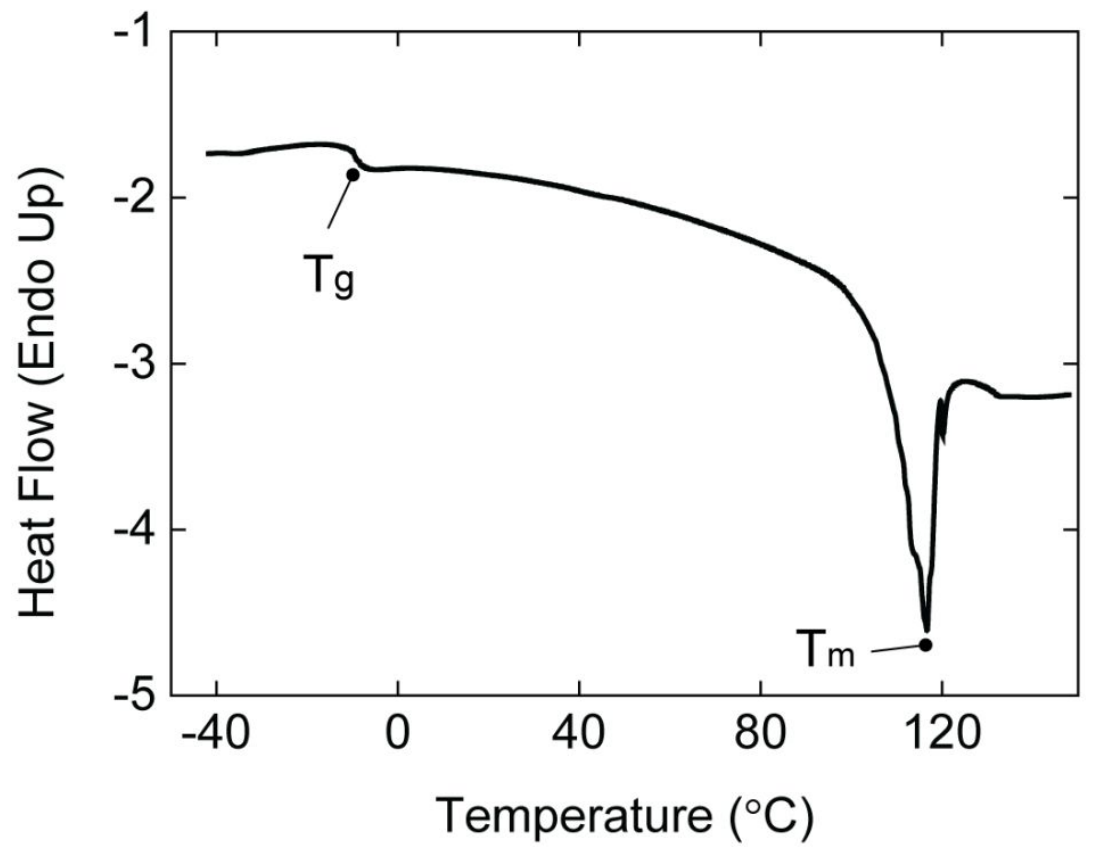

Figure S13. DSC data of the PTEO-LiTFSI (10 wt $\%$ ) in the temperature range of -40 to $150^{\circ} \mathrm{C}$. The glass transition temperature and the melting point of PTEO-LiTFSI were around $-8^{\circ} \mathrm{C}$ and $110^{\circ} \mathrm{C}$, respectively.

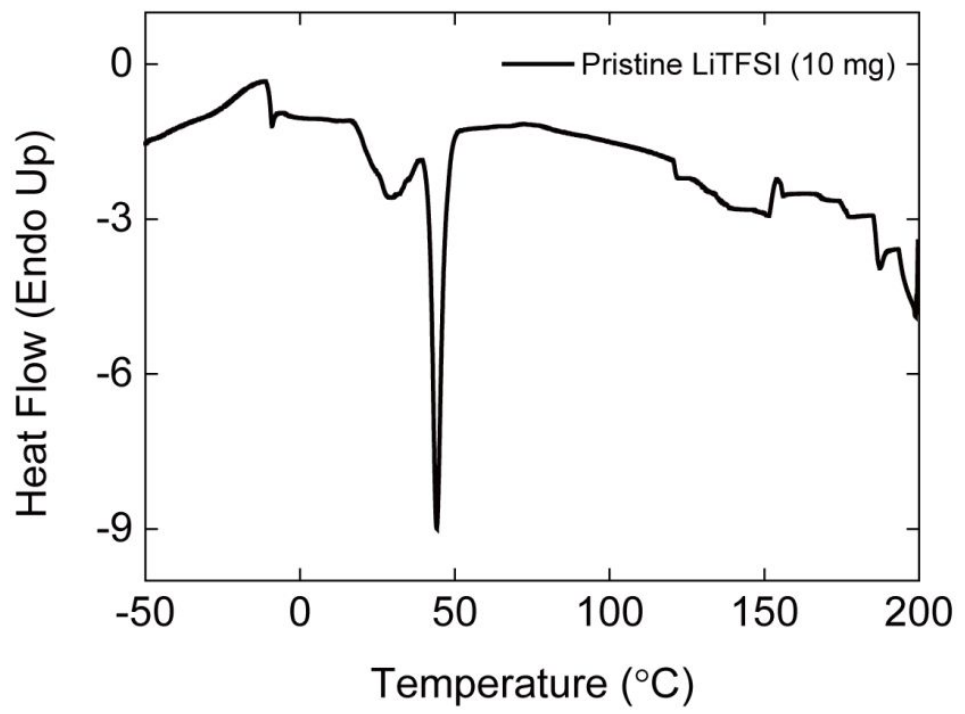

Figure S14. DSC data of the pristine LiFTSI $(10 \mathrm{mg})$ in the temperature range of -50 to $200^{\circ} \mathrm{C}$.

\section{$\underline{\text { References }}$}

[1] Y. Joo, V. Agarkar, S. H. Sung, B. M. Savoie, B. W. Boudouris, Science 2018, 359, 1391.

[2] C. Chang, J. Zhu, Z. Zhang, N. Zhou, Z. Cheng, X. Zhu, Polymer 2010, 51, 1947.

[3] T. Endo, K. Takuma, T. Takata, C. Hirose, Macromolecules 1993, 26, 3227. 